\title{
Fast Response Fresnel Liquid Crystal Lens for 2D/3D Autostereoscopic Display
}

\author{
Yi-Pai Huang*b ${ }^{\mathrm{b}}$, Chih-Wei Chen ${ }^{\mathrm{a}}$, Yi-Ching Huang ${ }^{\mathrm{a}}$ \\ ${ }^{\mathrm{a}}$ Department of Photonics \& Institute of Electro-Optical Engineering, ${ }^{\mathrm{b}}$ Display Institute, \\ National Chiao Tung University, Hsinchu, Taiwan (R.O.C.), 30010
}

\begin{abstract}
The fast response Fresnel liquid crystal lens with multiple electrodes structure had been proposed and optimized. The multi electrodes in the new Fresnel LC lens were placed at both inner surfaces of LC cell. By supplying specific operating voltages on each electrode, the optimized Fresnel LC lens was closed to the ideal Fresnel lens. The experiment results indicated that the Fresnel LC lens not only performed fast switching rate without over-drive method, but also had the advantage of low operating voltage. Furthermore, by using overdriving method, the response time could be further reduced but just need 15 volts in a short time. It meant that a fast switching between $2 \mathrm{D}$ and $3 \mathrm{D}$ image display could be obtained by switching Fresnel LC lens on and off. In the end, the fast switching display was also demonstrated.
\end{abstract}

Keywords: Liquid Crystal Lens, Fresnel Lens, Three Dimensional Display, Autostereoscopic Display

\section{INTRODUCTION}

Recently, many researchers invest in developing 3D display technology to produce more natural images from displays. For autostereoscopic display, such as holographic type ${ }^{1}$, volumetric type ${ }^{2}$, and multiplexed-2D type ${ }^{3}$, the observers can perceive 3D images without extra components. Now, the multiplexed-2D method has been widely used in 3D application due to its easy implementation property. However, the technologies such as fixed parallax Barrier ${ }^{4}$ and lenticular lens arrays $^{5,6}$ cause image degradation when displaying 2D image. Therefore, to overcome the degradation of $2 \mathrm{D}$ image quality and also to show 3D image, many 2D/3D switchable displays ${ }^{7}$ have been proposed. For instance, by replacing the fixed lenticualar lens with the liquid crystal (LC) lens ${ }^{8-10}$, the 2D and 3D images can be shown by just switching the LC lens on and off ${ }^{1,12}$. When applying certain voltages, the local electric fields are formed, and then the non-uniformed electric field distribution causes the liquid crystal material reorient that the refractive index changes accordingly. Then, the phase difference is achieved and results in the change of light direction ${ }^{13}$. Base on the above concept, the switchable LC lenses are electrically controlled to modulate the emissive light from image pixels as the operating voltage applied, and the light pass through the LC layer directly as off-status.

Although the liquid crystal lens can supply 2D/3D images switching property, the slow on/off switching time is still a limitation because of the thick LC layer. Therefore, decreasing the switching time is needed. The LC response time can be further improved by reducing cell gap and over-driving according to the LC response time formula ${ }^{14}$. According to this concept, the Multiple Electrodes Driving Fresnel LC lens (MeD-Fresnel LC lens) ${ }^{15}$ with narrow cell gap and low operating voltage is proposed. By the multi-electrode structure and its controlling method, the liquid crystal is reoriented and has a Fresnel lens-like shape. Consequently, the proposed MeD-Fresnel LC lens not only reduces the response time, but also keeps the optical properties similar to conventional LC lens. Hence, the MeD-Fresnel LC lens with fast switching rate can be applied for fast 2D/3D switchable display. Furthermore, the driving voltage is much lower than conventional LC lens.

\section{PRINCIPLE OF THE PROPOSED DESIGN}

Based on this basic GRIN lens principle ${ }^{16}$, the LC lens proposed in this study was designed to own the index distribution of the LC lens. The refractive index difference $(\Delta \mathrm{n})$ between the center and the edge along the radial direction could be evaluated as Eq. $1^{17}$. Where the $r$ is the radius of LC lens, $d$ is the thickness of LC layer, and $f$ is the focal length. The refractive index difference $(\Delta \mathrm{n})$ is a function of parabolic curve. Hence, the LC lens could focus light effectively when the $\Delta \mathrm{n}$ is closer to ideal parabolic curve.

*boundshuang@mail.nctu.edu.tw; phone +886 3 571-2121 ext 52924; fax +886 3 573-7681

Advances in Display Technologies; and E-papers and Flexible Displays, edited by Karlheinz Blankenbach, Liang-Chy Chien, Sin-Doo Lee, Ming Hsien Wu, Proc. of SPIE Vol. 7956, 79560P

(c) 2011 SPIE · CCC code: $0277-786 \mathrm{X} / 11 / \$ 18 \cdot$ doi: $10.1117 / 12.880032$

Proc. of SPIE Vol. $795679560 P-1$ 


$$
\Delta n=\frac{r^{2}}{2 d f}=\alpha \cdot r^{2}
$$

The multi-electrically Driven Liquid crystal (MeD-LC) lens structure ${ }^{12}$ proposed before was shown in Fig.1 (a). For proposed MeD-LC lens, the outer electrode made the electric field distribution smooth and continuous in the LC cell by applying certain voltages on each corresponding electrodes. Thus the refractive index distribution was close to the ideal curve and then built a good lens-like shape. However, the operating voltage had to be high to induce a strong electric field across the LC layer due to the thick glass which was sandwiched between electrode layer and LC layer. So the large operating voltage is also a key issue for application.

In order to reduce the operating voltage, the electrode layer must put inside LC layer. From Fig.1 (b), if the electrode layer was inside the cell, the electric field could be obtained easy just by small operating voltage. However, the discrete electric field would appear due to the grating structure. The un-uniform electric field made the light is focused badly. Nevertheless, from the concept of Fresnel lens ${ }^{17,18}$ which was proposed for years, the amount of material could be reduced by breaking the traditional lens into a set of different prisms but it still kept the same optical properties. Therefore, combining the concepts of inner-electrode MeD-LC lens and Fresnel lens, the new LC lens structure called Multiple Electrodes Driving Fresnel Liquid Crystal (MeD-Fresnel LC) lens was proposed and the scheme of concept was also shown in Fig. 2. The discrete electric field distribution of inner-electrode MeD-LC lens was useful for building Fresnel LC lens because of the Fresnel lens was a combination of a set of discrete prisms. Hence, the new MeD-Fresnel LC lens with low operating voltage and narrow cell gap was obtained.

\section{Outer Electrode}
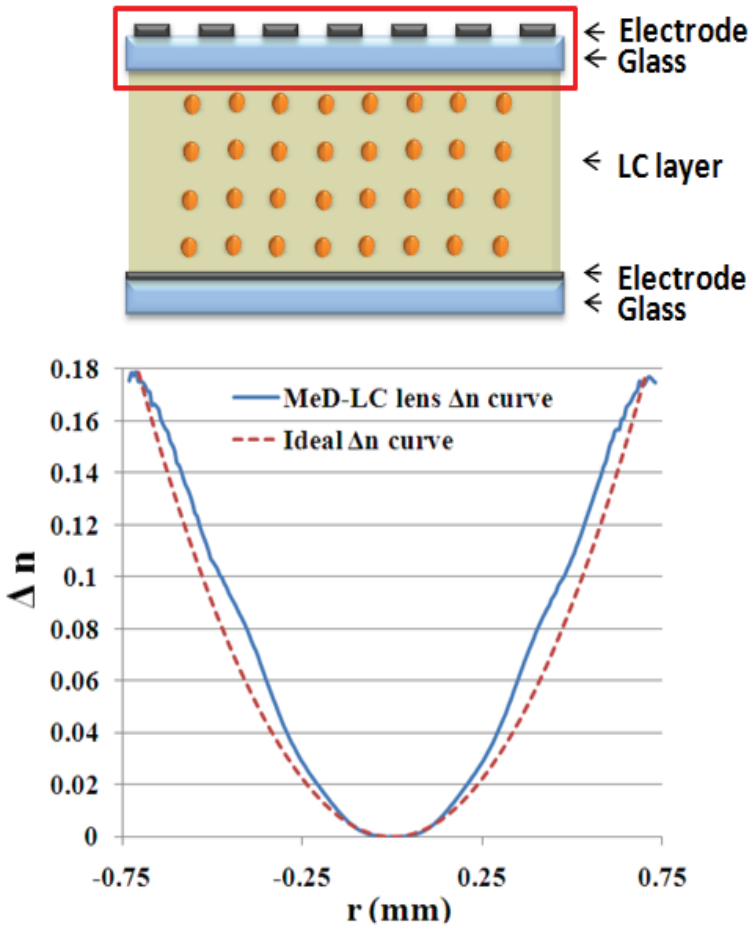

(a)

\section{Inner Electrode}
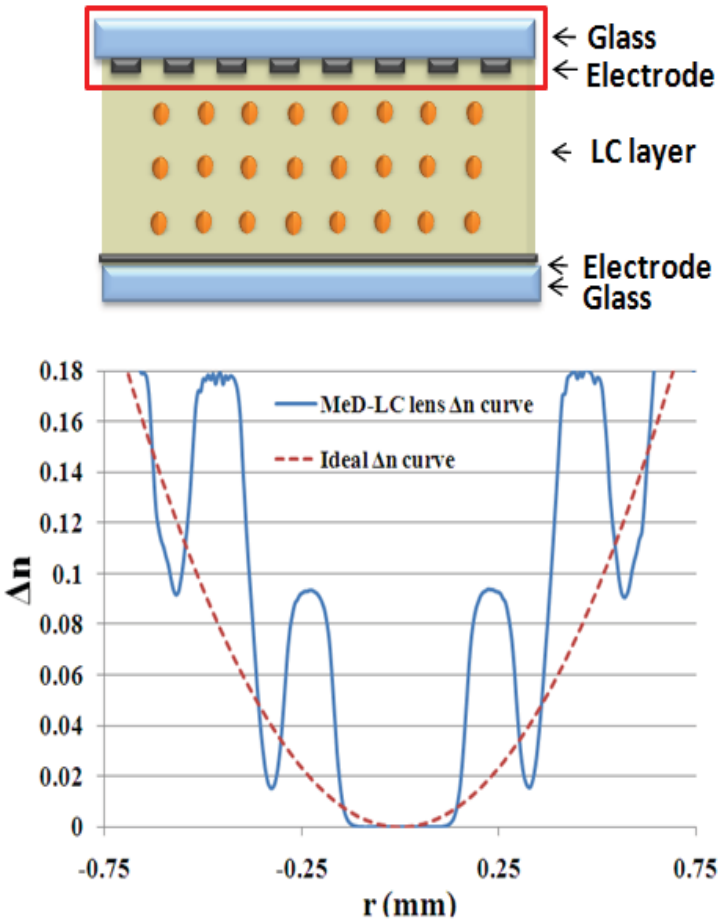

(b)

Figure 1. Refractive index difference $(\Delta \mathrm{n})$ distribution of the MeD-LC lens by using (a) outer electrode structure and (b) inner electrode structure 


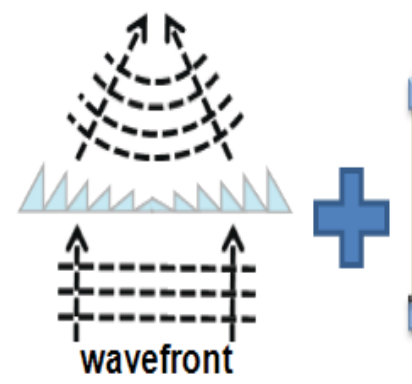

\section{Fresnel lens}

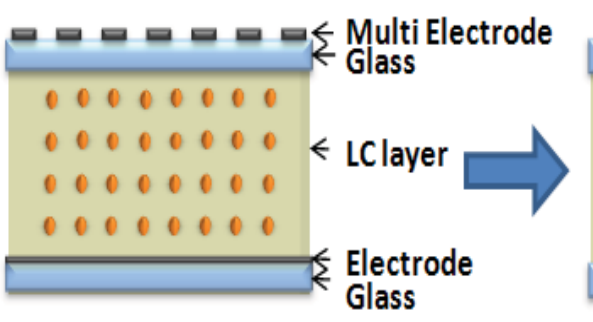

\section{MeD-LC lens}

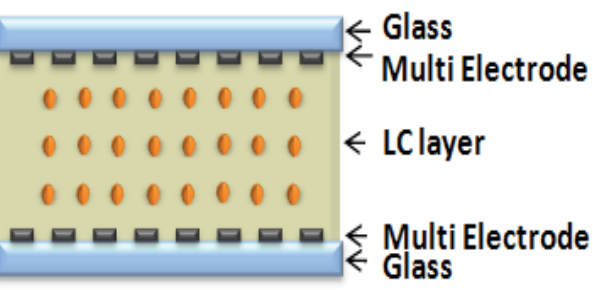

MeD-Fresnel LC lens

Figure 2. Concept of MeD-Fresnel LC lens : Fresnel lens + MeD-LC lens = MeD-Fresnel LC lens

\section{EXPERIMENT}

According to the simulation result presented in the above section, the experimental results were showed below. The results proved that our design had low operating voltage and fast response time compare to the traditional LC lens. Finally, the crosstalk phenomenon of the MeD-Frensel LC lens and demonstration were also shown. The prototype of MeD-Fresnel LC lens was fabricated and was measured as indicated in Fig. 3. Each MeD-Fresnel LC lens had 24 electrodes at both top and bottom substrates inside the LC cell that could be applied operating voltage individually to form different Fresnel prisms.

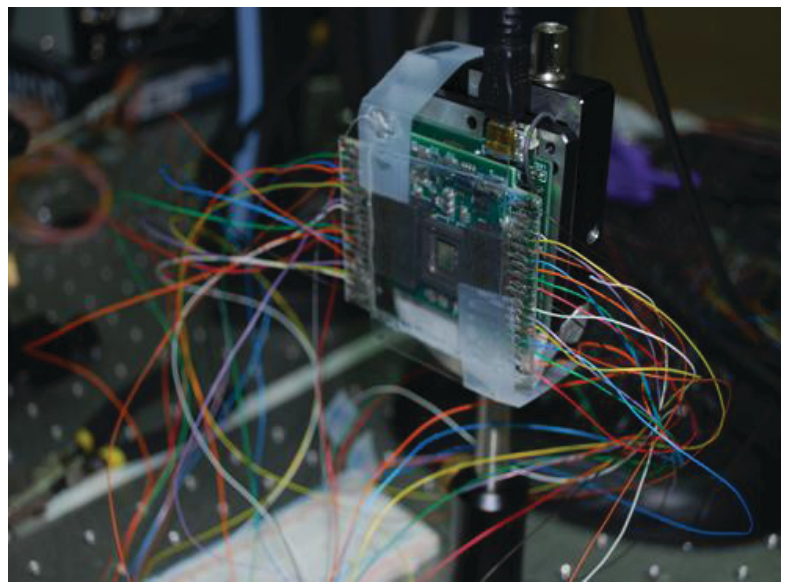

Figure 3. Prototype of MeD-Fresnel LC lens

To check the focusing ability of the MeD-Fresnel LC lens, the specific voltages were applied on each electrode. The incident light was He-Ne laser whose wavelength was around $632.8 \mathrm{~nm}$. The CCD was placed at the focal plane of the MeD-Fresnel LC lens to measure the light intensity distribution. The captured images of without applying voltages (unfocusing status) and with applying voltages (focusing status) were shown in Fig. 4 (a) and (b). Furthermore, the measured light intensity distribution was also shown in Fig. 4(c). The green region in Fig. 4(c) indicated the width of one MeD-Fresnel LC lens. The red line (dotted line) and blue line (solid line) indicated the incident light distributions when focusing and after focusing respectively. This result showed that the light was focused effectively by the MeD-Fresnel LC lens. Besides, the beam size can be judged by the full width at half maximum (FWHM), which was also used to compare the focusing ability. The smaller beam size represented a better focus ability. The FWHM of our design was about 59um, which was better than the traditional LC lens ( 80um). This result showed that the MeD-Fresnel LC lens with multi-electrode at both top and bottom substrates inner the LC cell made incident light be focused effectively. 


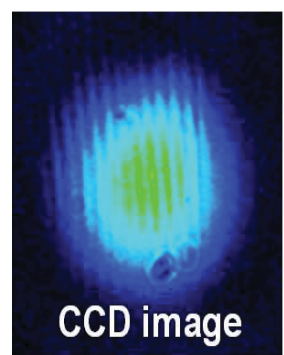

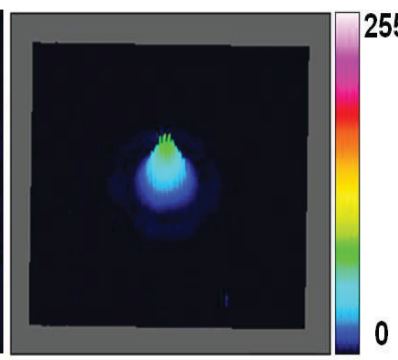

Before Focus

(a)
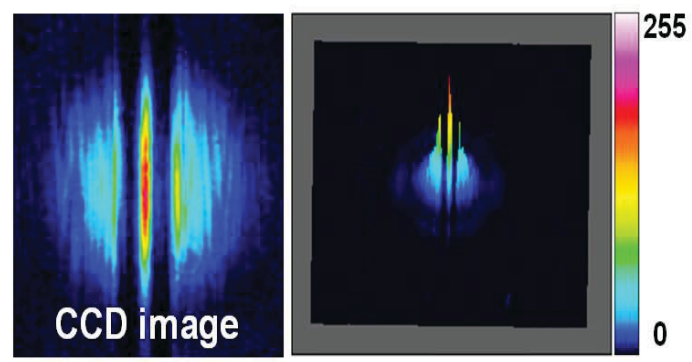

After Focus

(b)

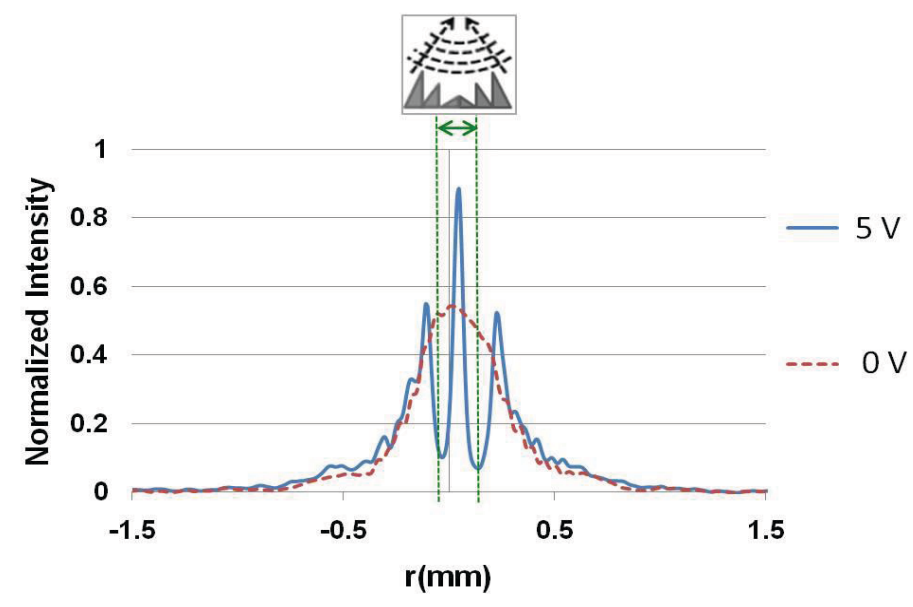

(c)

Figure 4. (a)The captured image of MeD-Fresnel LC lens before applying voltage, (b) the one after applying voltage , and (c) the measured power intensity at focal plane

In addition, the focus length was about $1 \mathrm{~mm}$ when the driving voltage was about 5 volts, and its frequency was 1 $\mathrm{KHz}$ under operation. Compared to the operating voltage of MeD-LC lens $(\sim 30$ volts, $1 \mathrm{KHz})$ for the same focal length, the proposed new structure had much lower operating voltage than traditional one.

To calculate the switching rate of the MeD-Fresnel LC lens, the CCD which could capture images sequentially by $0.2 \mathrm{~s}$ per frame was used. Besides, in LCD applications, the overdrive ${ }^{19}$ method was used to reduce the response time of LC cell. By applying a large voltage (pulse type) through the electrodes first, the LC molecules rotated in a very short time and then the desired operating voltage signal was applied to stabilize the focusing ability. Therefore, applying the overdrive method on the MeD-Fresnel LC lens, a pulsed voltage (3 times of the desired operating voltage) was applied to the $\mathrm{LC}$ cell. And the desired operating voltage signal $\left(5 \mathrm{~V}_{\mathrm{rms}}\right)$ was also applied following. The result was got and was shown in Fig.5. From the result, with the over-drive method, the switching rate of the MeD-Fresnel LC lens was reduced effectively within $0.2 \mathrm{~s}$. Comparing with conventional MeD-LC lens, the switching rate of MeD-Fresnel LC LENS was much less than conventional one which switching rate still took about $6 \sim 10$ seconds. 


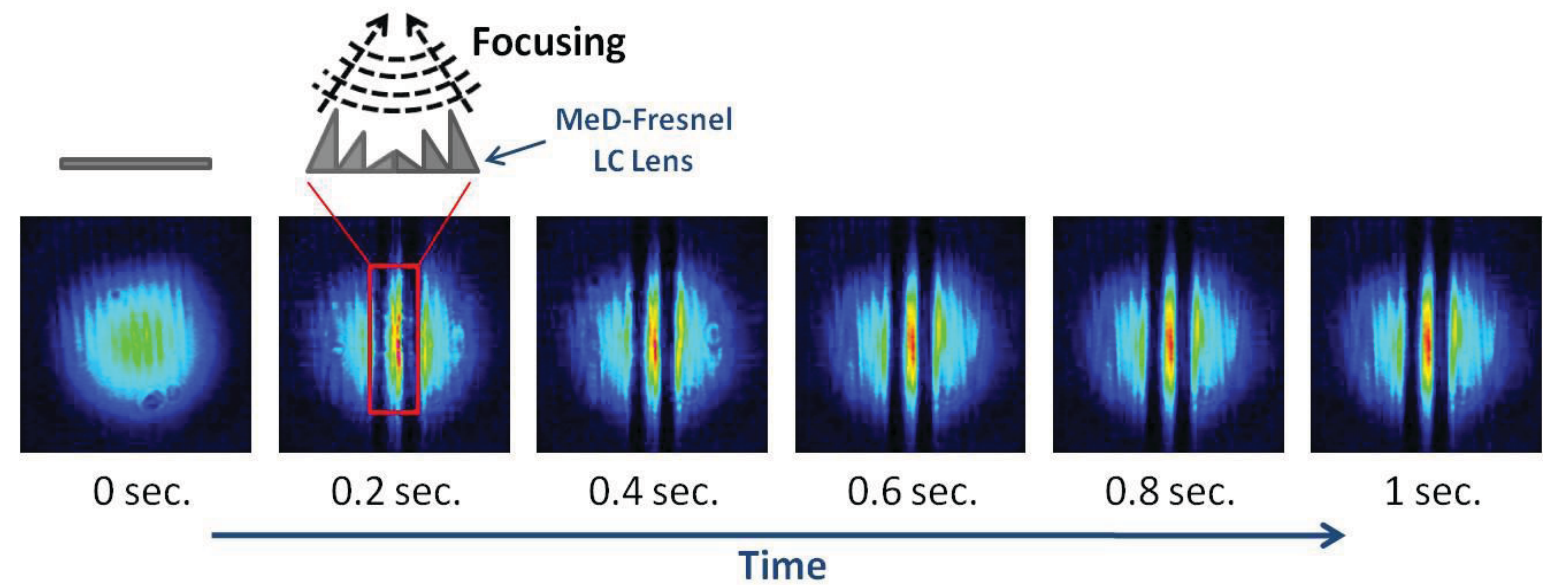

Figure 5. Sequential focusing images of the MeD-Fresnel LC lens with over-drive method. It indicated that the response time was around $0.2 \mathrm{~s}$.

In 3D displays, crosstalk could be defined as the leakage of left eye image to the right eye and right eye image to the left eye ${ }^{3}$. If the crosstalk was too high, human couldn't perceive two different images that no 3D sensation, as well as the uncomfortable feeling will be caused. The crosstalk phenomenon could be minimized when the images projected to the exact eyes. By ray-tracing method, the horizontal light intensity distribution of each viewing zone was simulated. The distance between each view was around $65 \mathrm{~mm}$ which identified with eye distance at the viewing distance $(\sim 1 \mathrm{~m})$, After calculating by Eq. 2, the crosstalk by using the MeD-Fresnel LC lens was obtained and the value was around $29 \%$, which was similar the MeD-LC lens (about 24\%) but lower than the double electrode LC lens (about 42\%). The results demonstrated that 3D display with the MeD-Fresnel LC lens could get the similar performance of 3D image as using traditional MeD-LC lens, but much better than the conventional double electrode LC lens.

$$
\text { Crosstalk }=\frac{I_{\min }}{I_{\max }+I_{\min }} \times 100 \%
$$

Finally, the prototype of 2D/3D switchable display with the proposed MeD-Fresnel LC lens was also demonstrated. The 2D image and 3D image were observed and were also shown in Fig. 6. The MeD-Fresnel LC lens was operated only 5 volts which was within the range of normal LC driving condition thus it was much easy for implementation.

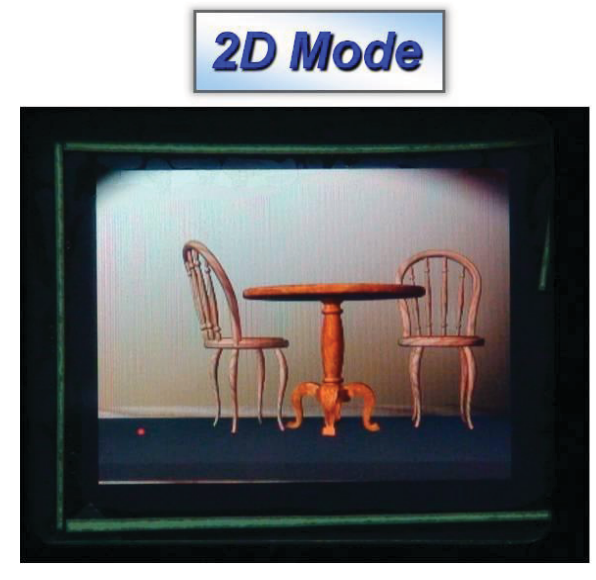

(a)

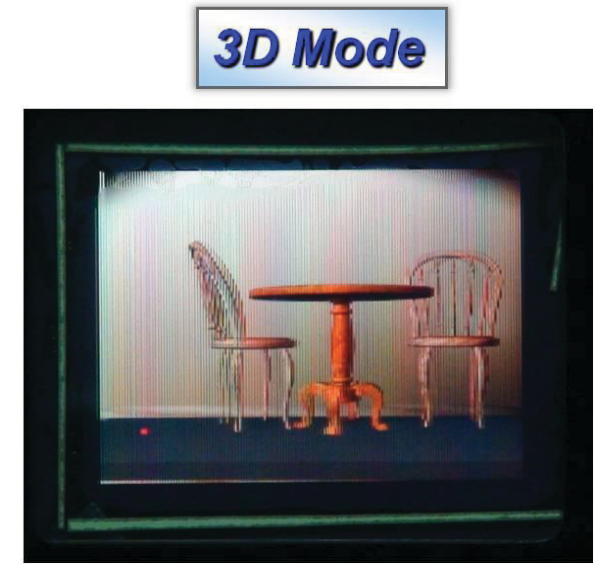

(b)

Figure 6. Snapshots of (a) 2D and (b) 3D images from the proposed 2D/3D switchable display 


\section{CONCLUSIONS}

A novel active device, Multiple Electrodes Driving Fresnel LC (MeD-Fresnel LC) lens was proposed. The simulation and experiment results indicated that a good Fresnel lens-like distribution device with multiple transparent electrodes structure at both top and bottom substrates inside the LC layer could be achieved. The MeD-Fresnel lens had a fast switching rate (less than 1s) without using over-drive method as well as the switching time could be further reduced to $0.2 \mathrm{~s}$ by using over-drive method. Compare to the switching time of the traditional MeD-LC lens $(\sim 4 \sim 10 \mathrm{~s})$, the response time had been further improved. Besides, the operating voltage $(\sim 5$ volts $)$ was much lower than the MeD-LC lens $(\sim$ 30 volts) that the proposed lens was much suitable for application. In addition, the crosstalk was also calculated and the value was around $29 \%$. Therefore, the fast switching between $2 \mathrm{D}$ and 3D image with the proposed structure was

demonstrated and was obtained through switching the MeD-Fresnel LC lens on and off.

\section{ACKNOWLEDGEMENT}

This work was partially supported by National Science Concil and ITRI, Taiwan, for Academic Projects No.NSC982220-E-009-019, and No. 98-EC-17-A-01-S2-0007. We also would like to thank Mr. B. W. Xiao, Mr. Y. H. Pai, Mr. K. J. $\mathrm{Hu}$ and Mr. J. F. Huang for their valuable support.

\section{REFERENCES}

[1] B. Javidi and E. Tajahuerce, "Three dimensional object recognition using digital holography," Opt. Lett. 25, 610-612 (2000).

[2] C. Myungjin and B. Javidi, "Free View 3-D Visualization of Occluded Objects by Using Computational Synthetic Aperture Integral Imaging," IEEE J. Display Technol. 5, 345-349 (2009).

[3] N. Holliman, [3D Display System] (2005).

[4] G. J. Woodgate, J. Harrold, A. M. S. Jacobs, R.R. Moseley, and D. Ezra, "Flat-panel autostereoscopic displays: characterization and enhancement," Proc. SPIE 3957, 153-164 (2000).

[5] C. V. Berkel and J. A. Clarke, "Characterization and optimization of 3D-LCD module design," Proc. SPIE 3012, 179-187 (1997).

[6] S. J. Young and B. Javidi, "Three-dimensional image methods based on multi-view images," IEEE J. Display Technol. 1, 125-140 (2005).

[7] J. Flack, J. Harrold, G. J. Woodgate, "A prototype 3-D mobile phone equipped with a next generation autostereoscopic display," Proc. SPIE 6490, 1-12 (2007).

[8] S. Sato, "Liquid-crystal lens-cells with variable focal length,” Jpn. J. Appl. Phys.18, 1679-1684 (1979).

[9] H. W. Ren, Y. H. Fan, S. Gauza, and S.T. Wu, “Tunable-Focus Cylindrical Liquid Crystal Lens,” Jpn. J. Appl. Phys.43, 652-653 (2004).

[10]H. W. Ren, D. W. Fox, B. Wu, and S. T. Wu, "Liquid crystal lens with large focal length tunability and low operating voltage," Opt. Express 15, 11328-11335 (2007).

[11] H. K. Hong, S. M. Jung, B. J. Lee, and H. H. Shin, "Electric-field-driven LC lens for 3D/2D autostereoscopic display”, J. Soc. Inf. Disp.17,399-406 (2009).

[12] Y. P. Huang, L. Y. Liao, and C. W. Chen, "2-D/3-D switchable autostereoscopic display with multi-electrically driven liquid-crystal (MeD-LC) lenses, ”J. Soc. Inf. Disp.18, 642-646 (2010).

[13] S.T. Kowel, D. S. Cleverly, and P. G. Kornreich, "Focusing by electrical modulation of refraction in a liquid crystal cell," Applied Optics 23, 278-289 (1984).

[14] S. T. Wu and D. K. Yang, [Reflective Liquid Crystal Displays], Wiley (2001).

[15] C. W. Chen, Y. C. Huang, and Y. P. Huang, "Fast Switching Fresnel Liquid Crystal Lens for Autostereoscopic 2D/3D Display", SID Int. Symp. Digest Tech. Papers 41, 425-428 (2010).

[16]E. Hecht, [Optics], Addison Wesley, (2002).

[17] J. W. Goodman, [Introduction to Fourier Optics], McGraw-Hill, (1968).

[18]A. Davis and F. Kuhnlenz, "Optical Design using Fresnel Lenses-Basic Principles and some Practical Examples," Optics \& Photonic 4, 52-55 (2007).

[19]L. Y. Liao, P.Y. Shieh, and Y. P. Huang, "Marginal electrodes with over-drive method for fast response liquid crystal lens applications," SID Int. Symp. Digest Tech. Papers 41 1766-1769 (2010). 